\title{
Right Atrial Size and Function to predict Left Atrial Voltage Defined Fibrosis in Patients with Long-standing Persistent Atrial Fibrillation
}

\author{
Radoslaw Kiedrowicz ${ }^{1}$, Maciej Wielusiński ${ }^{1}$, Andrzej Wojtarowicz ${ }^{2}$, and Jaroslaw \\ Kazmierczak $^{1}$ \\ ${ }^{1}$ Pomeranian Medical University in Szczecin \\ ${ }^{2}$ Department of Cardiology, Pomeranian Medical University, Szczecin, Poland
}

July 20, 2020

\begin{abstract}
Background: Right atrial (RA) size and function are not well described in long-standing persistent AF (LSPAF) patients, nor their value as a predictor for the left atrial (LA) voltage-defined fibrosis. Methods: An evaluation was made as to whether echocardiography determined RA length, planimetered area, volume, emptying fraction, stroke volume, expansion index and calculated derivates among the LSPSAF population predict LA low voltage areas (LVA) acquired with high-density and highresolution bipolar voltage mapping using the CARTOß3 system. Results: 142 patients aged 63 (58-67) years old, 117 males, were enrolled in our study. LVA were detected in $54 \%$ of the patients.Severe global LVA burden was present in $15 \%$ of the patients, whereas $30 \%$ of the patients presented a disseminated pattern of remodelling. We have shown that (1) the majority of the study population (76\%) presented enlarged RA, however RA volumes were larger than LA volumes in the minority of cases (2) RA enlargement had a positive correlation with the presence of mild-to-moderate tricuspid regurgitation, left ventricular hypertrophy, LA enlargement, LA area and volume (3), none of the RA indices were associated with the prediction of absolute LVA or advanced LA fibrotic remodelling, although patients with severe LVA burden presented longer RA length and a larger area than comparing to patients with less advanced remodelling pattern. Conclusions: It was found that RA enlargement and decreased RA function, common in LSPSAF patients, do not translate to the presence of voltage-derived LA fibrotic remodelling
\end{abstract}

\section{TITLE}

Right Atrial Size and Function to predict Left Atrial Voltage Defined Fibrosis in Patients with Long-standing Persistent Atrial Fibrillation

\section{RUNNING HEAD}

Right Atrial Indices in Persistent AF

\section{AUTHORS}

Radoslaw Marek Kiedrowicz MD, PhD. Pomeranian Medical University, Cardiology Department, Powstancow Wlkp. 72, 70-111 Szczecin, Poland ; email: radkied@wp.pl ; phone: +48509395560 ; fax: +48914661379 (Corresponding Author)

Maciej Wielusinski MD. Pomeranian Medical University, Cardiology Department, Szczecin, Poland

Andrzej Wojtarowicz MD, PhD. Pomeranian Medical University, Cardiology Department, Szczecin, Poland 
Jaroslaw Kazmierczak MD, PhD, Professor of Cardiology. Pomeranian Medical University, Cardiology Department, Szczecin, Poland

\section{FUNDING}

This work was supported by the Minister of Science and Higher Education [Regional Initiative of Excellence 002/RID/2018/19]

\section{ABSTRACT}

Background: Right atrial (RA) size and function are not well described in long-standing persistent AF (LSPAF) patients, nor their value as a predictor for the left atrial (LA) voltage-defined fibrosis.

Methods: An evaluation was made as to whether echocardiography determined RA length, planimetered area, volume, emptying fraction, stroke volume, expansion index and calculated derivates among the LSPSAF population predict LA low voltage areas (LVA) acquired with high-density and high-resolution bipolar voltage mapping using the CARTO $\cap 3$ system.

Results: 142 patients aged 63 (58-67) years old, 117 males, were enrolled in our study. LVA were detected in $54 \%$ of the patients. Severe global LVA burden was present in $15 \%$ of the patients, whereas $30 \%$ of the patients presented a disseminated pattern of remodelling. We have shown that (1) the majority of the study population (76\%) presented enlarged RA, however RA volumes were larger than LA volumes in the minority of cases (2) RA enlargement had a positive correlation with the presence of mild-to-moderate tricuspid regurgitation, left ventricular hypertrophy, LA enlargement, LA area and volume (3), none of the RA indices were associated with the prediction of absolute LVA or advanced LA fibrotic remodelling, although patients with severe LVA burden presented longer RA length and a larger area than comparing to patients with less advanced remodelling pattern.

Conclusions: It was found that RA enlargement and decreased RA function, common in LSPSAF patients, do not translate to the presence of voltage-derived LA fibrotic remodelling

\section{KEYWORDS}

Atrial Fibrillation; Long-standing Persistent Atrial Fibrillation; Fibrosis; Low Voltage Areas; Right Atrium

\section{Introduction}

Low voltage areas (LVA) in the left atrium (LA) are well documented in electrophysiology studies and reported as an arrythmogenic substrate operating in atrial fibrillation (AF) and suggested as fibrotic areas [1]. To date, there has been limited research evaluating the predictors of the LVA among long-standing persistent AF (LSPAF) patients. Recently, it was established that LA size and function do not correlate with LVA burden in this population [2]. The relationship between the LVA documented in the LA and right atrial (RA) size and function remains unknown. Moreover, in contrast to the LA, RA structure and function are not as well described in patients with LSPSAF. In this study we aimed to determine RA parameters among LSPSAF population and hypothesised that echocardiography derived RA indices may be helpful to detect LA LVA acquired with high-density and high-resolution bipolar voltage mapping in addition to LA parameters.

\section{Methods}

\section{Study population}

Consecutive LSPAF patients referred to our centre for RF point-by-point catheter ablation were prospectively enrolled. The patients with a history of AF ablation procedure, any cardiac surgery, severe valvular disease, previous myocarditis or pericarditis, known or suspected pulmonary hypertension, atrial septal defect and those who were not able to maintain sinus rhythm during LA mapping were excluded. All patients underwent 
an electrophysiological study and ablation under the approval of a local institutional committee on human research after providing written informed consent.

\section{Echocardiography examination}

All patients had well-controlled heart rate and underwent transthoracic and transoesophageal echocardiography prior to the ablation using a Vivid E9 ultrasound system (GE Vingmed Ultrasound AS). The following variables were prospectively measured during $\mathrm{AF}$ as an average of five cardiac cycles and indexed to the body surface area: LA anteroposterior diameter, RA and LA maximum and minimum length (long axis) and planimetered area and volume. All measurements were performed by a single experienced physician using well established criteria [3]. RA and LA contractile and reservoir functions were analysed calculating the following indices and expressed as percentage where appropriate: (1) emptying fraction (EF), by dividing the difference between maximum and minimum volume by maximum volume (2) stroke volume (SV), as the difference between maximum and minimum volume (3) expansion index (EI), by dividing the difference between maximum and minimum volume by minimum volume. The right to left atrial ratio of all determined parameters was then calculated and expressed as percentage. Moreover a biatarial volume index expressed as the sum of the right and left atrial indexed maximum and minimum volume and biatarial area index expressed as the sum of the right and left atrial indexed maximum and minimum planimetered area were calculated. Enlargement of the LA was defined with maximum indexed LA volume $>34 \mathrm{ml} / \mathrm{m}^{2}$ whereas enlarged RA with maximum indexed RA volume $>25 \mathrm{ml} / \mathrm{m}^{2}$ in men and $>21 \mathrm{ml} / \mathrm{m}^{2}$ in women. Left ventricular hypertrophy $(\mathrm{LVH})$ was defined on the basis of indexed ventricular mass calculated by system software $>$ $115 \mathrm{~g} / \mathrm{m}^{2}$ in men and $>95 \mathrm{~g} / \mathrm{m}^{2}$ in women [3]. Mitral (MR) and tricuspid (TR) regurgitation was assessed according to guidelines [4].

\section{Detection of LVA}

The mapping protocol is described in detail elsewhere [2]. Briefly, following pulmonary vein isolation (PVI) and cardioversion, all patients underwent high density-high resolution LA bipolar voltage mapping [2959 (2212-3143) points per map] during coronary sinus pacing using the CARTOß3 system (Biosense-Webster, BW) with a Pentaray catheter (BW) acquired with a CONFIDENSE module (BW). To ensure detailed mapping, the distance filling threshold was set at $5 \mathrm{~mm}$, the tissue proximity filter was always enabled and only mapping sites that were within a distance of $5 \mathrm{~mm}$ from the acquired shell contributed to a voltage map. Further discrete mapping using a SmartTouch catheter (BW), which always covered less than $10 \%$ of the total LA surface area (TSA), at sites presenting inadequate Pentaray-tissue contact was performed if necessary. Electrograms were only accepted if a contact force was [?]6g. EGM amplitude [?]0.5mV was defined as normal and $<0.5 \mathrm{mV}$ as diseased tissue. All points presenting low voltage were visually inspected and those incorrectly annotated were deleted from the map. An extension of all the areas showing low voltage potentials at least $5 \mathrm{~mm}$ away from the ablation lesion set was measured with custom $\mathrm{CARTO}(\mathrm{r}) 3$ system software. The global LVA burden was calculated as a sum of the LVA and then expressed as a percentage of the TSA. The section of the PV inside ablation encirclement, LAA and an area adjacent to the fossa ovalis were excluded from the TSA calculations. The extent of global LVA burden $>20 \%$ of the TSA was arbitrarily considered as severe on the basis of an observation that all detected LVA can be easily ablated if it occupies less than $20 \%$ of the TSA[2]. The body of LA was segmented into 5 areas: septum, anterior, posterior, inferior and lateral wall. If LVA were identified within 3 out of $5 \mathrm{LA}$ segments it was considered a disseminated pattern of voltage-defined remodelling (Figure 1).

\section{Statistical Analysis}

Continuous data are presented as median and IQR, the categorical variables as values and percentages. Comparisons between groups were performed with the Mann-Whitney U-test, or the chi2 test, as appropriate. Multivariate stepwise forward logistic regression analyses was performed to isolate factors associated with the existence of LVA. Only variables with significant p-values on univariate analysis were included in the multivariate model. The correlation between variables was assessed using a Spearman rank test. Statistical significance was accepted at $\mathrm{p}$ value $<0.05$. The analysis was performed using Statistica software version 


\section{3 (StatSoft).}

\section{RESULTS}

One hundred and forty two patients aged 63(58-67) years old, 117 males (82\%), were enrolled in our study. The median continuous AF duration was 24(12-36) months, range from 12-204 months and median LA anteroposterior diameter was $47(45-50) \mathrm{mm}$, ranging from 31-63mm. Further detailed data on patient characteristics and echocardiographic findings are presented in Table 1.

LVA were detected in 54\% (77/142) of the patients. Severe global LVA burden was present in 15\% (21/142, $27 \%$ of the patients with LVA), whereas $30 \%$ of the patients $(42 / 142,55 \%$ of the patients with LVA) presented a disseminated pattern of remodelling. Twenty five percent of the patients (36/142) had documented LVA on the septum, $31 \%(44 / 142)$ on the anterior wall, $42 \%(59 / 142)$ on the posterior wall, $24 \%(34 / 142)$ on the inferior wall, and $10 \%(20 / 142)$ on the lateral wall.

No differences were found among RA dataset in patients presenting LVA and disseminated pattern of LVA remodelling. However, patients with severe LVA burden showed significantly longer RA maximum and minimum length, larger maximum and minimum RA indexed area and biatrial maximum and minimum area (Table 2).

Using an univariate logistic regression technique it was found that none of the RA indices were associated with the prediction of the LVA, severe LVA or a disseminated LVA pattern.

Further analysis revealed that RA maximum volume had a positive correlation with the presence of mildto-moderate TR $(\mathrm{r}=0.24, \mathrm{p}=0.0009)$ and $\mathrm{CHA}_{2} \mathrm{DS}_{2}$ VASc score [?] $(\mathrm{r}=0.22, \mathrm{p}=0.01)$. The presence of $\mathrm{RA}$ enlargement positively correlated with TR $(\mathrm{r}=0.23, \mathrm{p}=0.02)$ and $\mathrm{LVH}(\mathrm{r}=0.2, \mathrm{p}=0.02)$. Moreover both RA maximum volume and the presence of RA enlargement positively correlated with the presence of LA enlargement $(\mathrm{r}=0.2, \mathrm{p}=0.04)$, maximum LA volume $(\mathrm{r}=0.44, \mathrm{p}<0.0001)$ and area $(\mathrm{r}=0.43, \mathrm{p}<0.0001)$. Rightto-left maximum volume ratio $>100 \%$ had a positive correlation with TR $(\mathrm{r}=0.23, \mathrm{p}=0.016), \mathrm{LVH}(\mathrm{r}=0.2$, $\mathrm{p}=0.02)$, and an inverse correlation with female sex $(\mathrm{r}=-0.2, \mathrm{p}=0.035)$. Right-to-left maximum area ratio $>$ $100 \%$ had an inverse correlation with female sex $(\mathrm{r}=-0.2, \mathrm{p}=0.03)$. Biatrial maximum volume had a positive correlation with age $(\mathrm{r}=0.23, \mathrm{p}=0.026)$ and $\operatorname{LVH}(\mathrm{r}=0.37, \mathrm{p}<0.0001)$, whereas biatrial maximum area had a correlation with age $(\mathrm{r}=0.28, \mathrm{p}=0.002), \mathrm{LVH}(\mathrm{r}=0.37, \mathrm{p}<0.0001), \mathrm{CHA}_{2} \mathrm{DS}_{2}$ VASc score $[?] 4(\mathrm{r}=0.24$, $\mathrm{p}=0.01)$ and inversely correlated with BMI $(\mathrm{r}=-0.34, \mathrm{p}<0.0001)$. No correlations between RA features and $\mathrm{AF}$ duration along with concomitant diseases other than those included in the $\mathrm{CHA}_{2} \mathrm{DS}_{2} \mathrm{VASc}$ score were found.

\section{DISCUSSION}

LA remodelling is a common response to AF, manifesting as increased size and reduced function, deteriorating with increasing AF burden [5]. RA structure and function were not thoroughly investigated in patients with LSPSAF than compared to LA. Moreover, data on the association between RA parameters and LVA burden seem to be lacking. Therefore this study aimed to describe echocardiography determined RA dataset among LSPSAF patients and identify if any of these could predict the existence of voltage-derived LA fibrotic remodelling.

We have shown that (1) the majority of the study population presented enlarged RA, however RA volumes were larger than LA volumes in the minority of cases (2) RA enlargement had a positive correlation with the presence of mild-to-moderate TR, LVH, LA enlargement, LA area and volume (3), none of the RA indices were associated with the prediction of the absolute LVA or advanced LA fibrotic remodelling, although patients with severe LVA burden presented longer RA length and a larger area comparing to patients with a less advanced remodelling pattern.

Many studies reported that voltage-defined LA remodelling increases with LA size [6-8] however not among LSPSAF patients [2]. It was suggested that the presence of functional LA enlargement due to AF persistence exclusively, seems not translate into atrial fibrosis. Three patterns of increased LA size in the LSPSAF 
population were proposed i) functional and at least partially reversible as a consequence of AF itself ii) secondary to other causes e.g. valvular regurgitation (primary or functional due to annular dilatation caused by $\mathrm{AF}$ ) iii) a combination of both [2].

In our study cohort, $76 \%$ of patients presented enlarged RA. In the vast majority of cases (83\%) RA indexed volumes were lower than LA volumes, in $11 \%$ it represented half of the LA volume. As RA maximum volume and the presence of RA enlargement correlated with the presence of LA enlargement, maximum LA volume and its area may suggest that increased RA size was the consequence of increased LA size in the context of $\mathrm{AF}$ persistence. It was previously reported that both LA and RA enlargement can develop as a consequence of $\mathrm{AF}$ in patients who had no evidence of significant structural or functional cardiac abnormalities other than AF [9]. This could explain why there was no association between RA size expressed in various parameters and LVA in our dataset. However, the presence of mild-to-moderate TR and LVH that correlated with $\mathrm{RA}$ enlargement and $\mathrm{CHA}_{2} \mathrm{DS}_{2}$ VASc score [?]4 that correlated with RA maximum volume could suggest that increased RA size was, at least in some cases, secondary to other scenarios than AF persistence. It is worth noting that cases where RA indexed volumes were higher than LA volumes also correlated with TR and LVH. RA enlargement is a common finding in patients with pulmonary arterial hypertension and atrial septal defect, though unlikely in our study group, and was found to be associated with the increased prevalence of AF [10]. Hypertensive LVH, very common in our cohort, is the known causative factor of diastolic dysfunction and has been shown to affect right-sided cardiac morphology and haemodynamics [11]. Therefore, it seems that increased RA size among LSPSAF patients is multifactorial.

The role of RA systolic (EF, SV) and diastolic (EI) function in the pathophysiology of AF persistence is yet to be analysed systematically. Moreover, due to the absence of established cut-off values [3]it is hard to assess RA function pattern. Accepting magnetic resonance-derived normative data [12] all patients presented severely decreased RA function. Larger RA volume index and lower RA EF have been shown to be a predictor of recurrence of AF after direct current cardioversion and were superior to LA parameters in one study [13]. In our study RA functional parameters were not associated with LVA. A clear interpretation of these findings remain unclear.

\section{CONCLUSIONS}

We have found that RA enlargement and decreased RA function, common in LSPSAF patients, do not translate to the presence of voltage-derived LA fibrotic remodelling.

\section{STUDY LIMITATIONS}

1) Whilst LSPSAF is considered more like a LA disease, to what extent the RA contributes to the role in the development and maintenance of AF remains unclear. Whether RA voltage-defined fibrosis, not assessed in our study, could improve risk stratification in LSPSAF patients should be analysed in future research investigations 2) The accuracy of LA voltage mapping might have been influenced by several factors, such as mapping following PVI or using voltage cut-off values $<0.5 \mathrm{mV}$. We cannot further exclude the fact that the overall LVA burden might have been altered due to the exclusion of patients not able to maintain sinus rhythm, presenting LAA thrombus or if another method of LVA detection had been applied 3) Women were underrepresented in the study

\section{CONFLICT OF INTEREST}

RMK has received fellowship support from BW. RMK, MW and JK have received educational support, travel grants, compensation for proctoring services from BW. AW declares no conflict of interest.

\section{REFERENCES}

Blandino A, Bianchi F, Grossi S, et al: Left Atrial Substrate Modification Targeting Low-Voltage Areas for Catheter Ablation of Atrial Fibrillation: A Systematic Review and Meta-Analysis. Pacing Clin Electrophysiol. 2017;40:199-212. 
Kiedrowicz R, Wielusinski M, Wojtarowicz A, Kazmierczak J: Predictors of the Voltage Derived Atrial Fibrosis in Patients with Long-Standing Persistent Atrial Fibrillation. Cardiol J doi: 10.5603/CJ.a2020.0069

Lang RM, Badano LP, Mor-Avi V, Afilalo J, et al: Recommendations for Cardiac Chamber Quantification by Echocardiography in Adults: An Update from the American Society of Echocardiography and the European Association of Cardiovascular Imaging. Eur Heart J Cardiovasc Imaging. 2015;16:233-70

Lancelotti P,Tribouilloy C, Hagendorff A, et al: Recommendations for the echocardiographic assessment of native valvular regurgitation: an executive summary from the European Association of Cardiovascular Imaging. Eur Heart J Cardiovasc Imaging 2013;14:611-44

Gupta DK, Shah AM, Giugliano RP, et al: Left atrial structure and function in atrial fibrillation: ENGAGE AF-TIMI 48. Eur Heart J. 2014; 35:1457-1465.

Huo Y, Gaspar T, Pohl M, et al: Prevalence and predictors of low voltage zones in the left atrium in patients with atrial fibrillation. Europace. 2018;20:956-962.

Kottkamp H, Berg J, Bender R, et al: Box Isolation of Fibrotic Areas (BIFA): A Patient-Tailored Substrate Modification Approach for Ablation of Atrial Fibrillation. J Cardiovasc Electrophysiol. 2016 Jan;27(1):22-30.

Huang D, Li JB, Zghaib T, et al: The Extent of Left Atrial Low-Voltage Areas Included in Pulmonary Vein Isolation Is Associated With Freedom from Recurrent Atrial Arrhythmia. Can J Cardiol. 2018 Jan;34(1):7379 .

Sanfilipo AJ, Abascal VM, Sheehan M, et al: Atrial Enlargement as a Consequence of Atrial Fibrillation. A Prospective Echocardiographic Study. Circulation 1990;82:792-7

Waligora M,TyrkaA, Miszalski-JamkaT, et al: Right Atrium Enlargement Predicts Clinically Significant Supraventricular Arrhythmia in Patients With Pulmonary Arterial Hypertension. Heart Lung 2018;4:237242

Cuspidi C, Negri F, Tadic MV, et al: Left Atrial Enlargement and Right Ventricular Hypertrophy in Essential Hypertension. Blood Press 2014;23:89-95

Maceira A, Sales JC, PrasadSK, Pennell DJ: Characterization of left and right atrial function in healthy volunteers by cardiovascular magnetic resonance. J Cardiovasc Magn Reson2016;18:64

Luong C, Thompson DJ, Bennett M, et al: Right atrial volume is superior to left atrial volume for prediction of atrial fibrillation recurrence after direct current cardioversion. Can J Cardiol. 2015; 31:29-35.

\section{AUTHOR CONTRIBUTIONS}

RMK: concept/design, data collection, data analysis/interpretation, drafting article, approval of article, statistics

MW: data collection, data analysis/interpretation, critical revision of article, approval of article

AW: data collection, data analysis/interpretation, critical revision of article, approval of article

JK: data analysis/interpretation, critical revision of article, approval of article, funding secured by

\section{FIGURE LEGENDS}

Figure 1. Left atrial (LA) distribution of low voltage areas (LVA) and examples of voltage maps of patients without LVA (A ), with disseminated pattern of voltage-defined remodelling as 3 out of 5 LA segments were affected (B ) and severe LVA burden $>20 \%$ of the total LA surface area $(\mathbf{C})$. All panels show anteroposterior and posteroanterior views of CARTO(r)3 LA shells acquired following pulmonary veins isolation and restoration of sinus rhythm with cardioversion. Color-coding is defined as follows: $<0.5 \mathrm{mV}=$ diseased atrial tissue (red), $>0.5 \mathrm{mV}=$ healthy atrial myocardium (pink). ant=anterior wall, sep=septum, post=posterior 
wall, inf=inferior wall, lat=lateral wall, $1=$ left atrial appendage, $2=$ right upper pulmonary vein, $3=$ right lower pulmonary vein, $4=$ left upper pulmonary vein, $5=$ left lower pulmonary vein

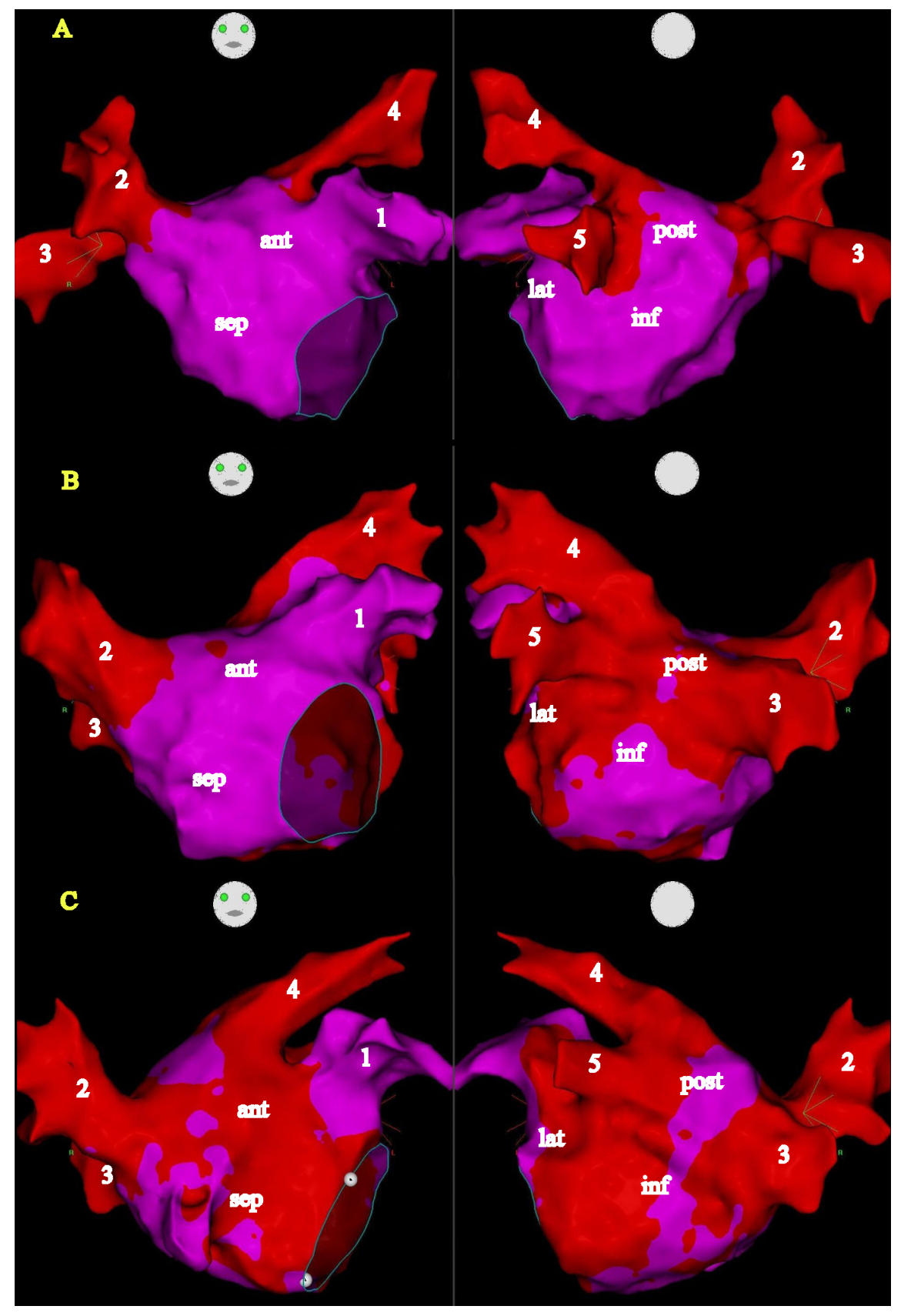

